Disponível em

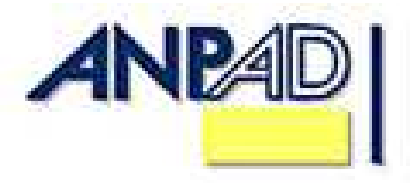

http://www.anpad.org.br/rac

RAC, Rio de Janeiro, v. 16, n. 5, art. 6, pp. 744-764, Set./Out. 2012

(cc) EY-No

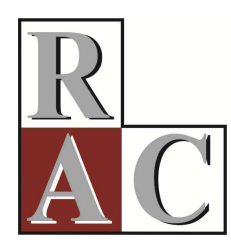

\title{
Bem-estar no Trabalho: Relações com Estilos de Liderança e Suporte para Ascensão, Promoção e Salários
}

\author{
Work well-being: Relationships with Leadership Styles and Support for Ascension, \\ Promotion and Salaries
}

Liliane Lima Sant'anna*

E-mail: lilianelimasantanna@gmail.com

Universidade de Brasília - UnB

Brasília, DF, Brasil.

Tatiane Paschoal

E-mail: tatipas@yahoo.com

Universidade de Brasília - UnB

Brasília, DF, Brasil.

Eliana Elisabete Moreira Gosendo

E-mail: gosendo@gmail.com

Faculdade de Tecnologia Senac Brasília, DF, Brasil.

\footnotetext{
* Endereço: Liliane Lima Sant'anna

Universidade de Brasília, Departamento de Administração, Campus Universitário Darcy Ribeiro, ICC Norte, Sala B1-576, Asa Norte, Brasília, DF, 70910-900.
} 


\title{
Resumo
}

O bem-estar tem sido apontado como fenômeno essencial para o funcionamento adequado e competitivo da organização. Este estudo testou a relação do suporte organizacional para ascensão, promoção e salários, e os estilos gerenciais com o bem-estar no trabalho. Essa variável contemplou o afeto positivo, o afeto negativo e a realização pessoal do trabalhador. O estudo foi conduzido em duas organizações privadas. Um total de 157 trabalhadores respondeu a um questionário composto pela Escala de Avaliação do Estilo Gerencial (Melo, 2004), pela Escala de Suporte Organizacional Percebido (Tamayo, Pinheiro, Tróccoli, \& Paz, 2000) e pela Escala de Bem-Estar no Trabalho (Paschoal \& Tamayo, 2008), todas validadas no Brasil. Os dados foram analisados por meio de correlações bivariadas e pela regressão múltipla padrão. Os resultados indicaram que tanto os estilos gerenciais quanto o suporte para ascensão, promoção e salários apresentam associações significativas com o bem-estar no trabalho. O suporte, por sua vez, apresentou as associações mais fortes com os fatores de bemestar, e foi seu principal preditor. Assim, quanto mais positiva é a percepção de suporte, maiores são o afeto positivo e a realização do trabalhador, e menor é o afeto negativo. Ações e práticas da organização parecem ser mais importantes para o bem-estar laboral do que comportamentos específicos de gestores.

Palavras-chave: afeto no trabalho; realização no trabalho; liderança; suporte organizacional; felicidade no trabalho.

\begin{abstract}
Well-being has been pointed out as an essential phenomenon for the adequate and competitive performance of organizations. This current study tested the relationships managerial styles and organizational support for ascension, promotion, and salaries have with work well-being. This variable includes the positive effects, the negative effects and personal fulfillment at work. The study was conducted in two private organizations. A total of 157 workers answered a survey composed by using the Evaluation Scale of Managerial Styles (Melo, 2004), Organizational Support Scale (Tamayo, Pinheiro, Tróccoli, \& Paz, 2000) and Work Well-being Scale (Paschoal $\&$ Tamayo, 2008), all of which have been validated in Brazil. The data was analyzed by means of bivariate correlation and standard multiple regression. The results indicate that managerial styles as well as support for ascension, promotion and salary have significant association with work well-being. The variable support presented stronger associations with well-being factors and it was also the most important predictor. The more positive the perception of support is, the greater the positive affect and worker fulfillment is and the lesser the negative affect is. Organizational actions and practices seem to be of utmost importance for work well-being, rather than specific behavior of managers.
\end{abstract}

Key words: effect at work; fulfillment at work; leadership; organizational support; happiness at work. 


\section{Introdução}

Nos últimos anos, a valorização das organizações tem sido associada aos seus ativos intangíveis, constituídos por recursos valiosos, raros e insubstituíveis, que garantem vantagem competitiva em longo prazo (Kayo, Kimura, Martin, \& Nakamura, 2006). Os ativos humanos constituem uma das categorias dos ativos intangíveis da organização, e envolvem o conhecimento, as habilidades, os talentos e a experiência dos seus integrantes (Barbosa \& Gomes, 2002). Segundo Oliveira e LimongiFrança (2005), as pessoas compõem os ativos intangíveis da organização e consistem em seus principais diferenciais competitivos.

Muitas organizações vêm divulgando um interesse em desenvolver estratégias para valorizar seu quadro de pessoal e estabelecer condições necessárias para o seu bom desempenho, sua satisfação e seu bem-estar. Tanto gestores quanto acadêmicos sugerem que ações e práticas organizacionais e gerenciais devam ser direcionadas para o desenvolvimento dos ativos humanos e o aumento da competitividade organizacional (Demo, 2008). Apesar disso, poucos estudos empíricos têm sido conduzidos de forma sistemática sobre os impactos das diferentes práticas e ações organizacionais nas experiências positivas dos trabalhadores. A maioria das pesquisas avalia as consequências negativas que o trabalhador pode vir a ter em decorrência de seu envolvimento com atividades laborais, como o estresse, a exaustão e o esgotamento (Warr, 2007).

O bem-estar, especialmente as experiências positivas do trabalhador, tem sido apontado como fenômeno essencial para o funcionamento adequado e competitivo da organização. Assim, torna-se relevante conhecer quais práticas, ações ou características da organização podem influenciar positivamente o bem-estar no trabalho.

De forma geral, a revisão da literatura indica que variáveis relacionadas ao trabalho e à organização influenciam diretamente um ou outro indicador de bem-estar (Warr, 2007). Apesar disso, como o enfoque das pesquisas geralmente recai sobre sintomas e experiências negativas do trabalhador, torna-se difícil sistematizar os achados e orientar os gestores sobre as práticas mais efetivas para possibilitar uma vivência positiva na organização. Muitos pesquisadores do bem-estar nas organizações focalizam exclusivamente, por exemplo, as estratégias para redução de impactos negativos do trabalho na saúde mental do trabalhador (Ferraz, Tavares, \& Ziberman, 2007).

Outra lacuna no campo do bem-estar no trabalho diz respeito à dificuldade de generalização dos resultados encontrados, pois os estudos são conduzidos com organizações e grupos de trabalhadores específicos, e raramente são replicados. Resultados de pesquisa de campo conduzida em uma organização pública, por exemplo, podem não representar o fenômeno em organizações privadas; resultados de pesquisa conduzida com funcionários de organizações formais podem não se aplicar a trabalhadores informais.

O objetivo geral desta pesquisa foi testar a relação de variáveis organizacionais percebidas com o bem-estar do trabalhador. Mais especificamente, foram testadas a relação do suporte organizacional para ascensão, promoção e salários com o bem-estar no trabalho e a relação dos estilos de liderança com o bem-estar no trabalho.

Para definição e operacionalização do bem-estar no trabalho, além do afeto negativo, foram incluídos o afeto positivo e a realização do trabalhador, ambos elementos claramente positivos do fenômeno. As variáveis independentes foram o suporte organizacional para ascensão, promoção e salários, e os estilos de liderança, que consistem em variáveis organizacionais e laborais percebidas pelo trabalhador.

A próxima seção apresenta o referencial teórico que guiou a pesquisa, incluindo uma breve revisão da literatura sobre bem-estar no trabalho, suporte organizacional e estilos de liderança, respectivamente. Em seguida, são apresentados o método adotado, os principais resultados e a 
discussão dos achados à luz da literatura revisada. Por fim, são destacadas as contribuições e as limitações da pesquisa, e indicadas questões para estudos futuros.

\section{Referencial Teórico}

A seguir, é apresentado o referencial teórico pertinente às variáveis abordadas no estudo: bemestar no trabalho, suporte organizacional para ascensão, promoção e salários, e estilos de liderança.

\section{Bem-estar no trabalho}

O campo do bem-estar tem sido marcado pela variedade de abordagens e modelos teóricos (Diener, 1984; Ryff \& Singer, 2008; Warr, 2007). As diferentes definições encontradas na literatura do bem-estar geral afetam os estudos sobre o bem-estar em dimensões específicas da vida. Como resultado da influência das correntes do bem-estar subjetivo (Diener, 1984) e do bem-estar psicológico (Ryff \& Singer, 2008), a literatura organizacional tem definido e operacionalizado o bem-estar no trabalho em termos hedônicos ou em termos de realização pessoal.

A abordagem hedônica refere-se ao bem-estar no trabalho como as emoções e humores vivenciados pelo trabalhador. O bem-estar seria maior quanto mais frequentes e intensas fossem as emoções e os humores positivos, e quanto mais essas experiências prevalecessem sobre o afeto negativo (Daniels, 2000; Ferreira, Silva, Fernandes, \& Almeida, 2008). O prazer e a satisfação com a vida são os elementos centrais da visão hedonista do bem-estar. A abordagem do bem-estar no trabalho como realização pessoal, por sua vez, enfatiza a percepção de desenvolvimento e expressão de potenciais individuais do trabalhador (Warr, 2007).

Diversos autores da literatura organizacional têm preferido incluir tanto os afetos quanto a realização pessoal nas definições de bem-estar (Ryan \& Deci, 2001; Warr, 2007). Segundo Waterman, Schwartz e Conti (2008), diferentes situações podem gerar experiências de bem-estar distintas, e tanto as emoções prazerosas quanto a percepção de realização são fundamentais para a compreensão do complexo fenômeno da felicidade ou do bem-estar pessoal.

Warr (2007) afirma que existem dois tipos importantes e complementares de bem-estar no trabalho. O primeiro corresponde a sentimentos de prazer vivenciados pelo trabalhador e se relaciona com a felicidade hedônica. O segundo tipo de bem-estar no trabalho refere-se a experiências denominadas de autovalidação. Quanto à felicidade hedônica, as emoções de prazer e a excitação combinam-se para determinar a tônica afetiva do indivíduo. Emoções de prazer, entusiasmo e conforto são representantes do afeto positivo e indicam alto bem-estar, enquanto emoções de desprazer, ansiedade e depressão representam o afeto negativo e indicam baixo bem-estar. Quanto à autovalidação, o desenvolvimento de atributos pessoais, a aplicação do próprio potencial e a expressão pessoal do trabalhador são seus aspectos constituintes (Warr, 2007).

Paschoal e Tamayo (2008), de forma compatível com as propostas de Waterman et al. (2008) e Warr (2007), conceituam o bem-estar no trabalho como a prevalência de emoções positivas neste, e a percepção do indivíduo de que, no seu trabalho, expressa e desenvolve seus potenciais e avança no alcance de suas metas de vida. Portanto, o bem-estar no trabalho inclui tanto aspectos afetivos (emoções e humores) quanto cognitivos (percepção de realização), e engloba os pontos centrais da abordagem hedonista e da realização pessoal. Em termos operacionais, o bem-estar no trabalho pode ser organizado em torno de três fatores: afeto positivo, afeto negativo e realização pessoal. Tais definição e operacionalização de bem-estar no trabalho são adotadas na presente pesquisa.

Em relação aos antecedentes do bem-estar do trabalhador, pesquisas têm sugerido que variáveis da organização podem ser mais importantes que variáveis pessoais. Estudos diversos indicam o papel preditor de variáveis, como oportunidades de controle e autonomia (Kelloway, Gottlieb, \& Barham, 
1999), conflito de papéis (Silva \& Ferreira, 2011), sobrecarga de trabalho (Silva \& Ferreira, 2011), uso de habilidades profissionais (Wilson, Dejoy, Vandenberg, Richardson, \& McGrath, 2004), percepção de justiça organizacional (Barsky \& Kaplan, 2007; Paz, Gosendo, Dessen, \& Godeville-Mourão, 2009), configurações de poder (Dessen \& Paz, 2010) e suporte organizacional (Paschoal, Torres, \& Porto, 2010).

Mesmo com a grande quantidade de estudos sobre antecedentes do bem-estar no trabalho, é difícil sistematizar os achados, pois a definição e a operacionalização do construto são variadas. Além disso, quando abordam elementos hedônicos do bem-estar, é mais comum encontrar pesquisas sobre as emoções negativas no trabalho. A dimensão da realização pessoal do trabalhador, por sua vez, quase nunca é mensurada (Paschoal et al., 2010).

Com base nas diversas lacunas do campo do bem-estar no trabalho, a presente pesquisa abordou o afeto positivo, o afeto negativo e a realização do trabalhador, e foi conduzida em duas empresas privadas. A seguir, são apresentadas as variáveis independentes do estudo.

\section{Suporte organizacional para ascensão, promoção e salários}

Segundo Eisenberger, Huntington, Hutchison e Sowa (1986) e Oliveira-Castro, Pilati e BorgesAndrade (1999), os trabalhadores formulam crenças sobre o quanto a organização valoriza e retribui seus esforços pessoais e se preocupa com seu bem-estar pessoal. Percepção de suporte organizacional é como tem sido denominado tal fenômeno. Basicamente, os trabalhadores realizam um processo de personificação da organização e passam a avaliar as ações dos agentes organizacionais como ações da própria organização (Eisenberger, Huntington, Hutchison, \& Sowa, 1986). Na definição do suporte organizacional, portanto, o agente das ações de retribuição consiste na própria organização, e não se refere a ações de gestores e agentes específicos.

Baseados no instrumento proposto por Eisenberger et al. (1986), Oliveira-Castro et al. (1999) desenvolveram uma medida de percepção de suporte organizacional para o Brasil. Os resultados do estudo indicaram que o construto poderia ser estruturado em torno de quatro fatores: gestão de desempenho; carga de trabalho; suporte material; práticas organizacionais de ascensão, promoção e salários. O primeiro fator, gestão de desempenho, refere-se à forma de gerenciamento do desempenho dos funcionários e envolve o tipo de estabelecimento de metas, a valorização de novas ideias, o conhecimento de dificuldades associadas à execução das tarefas e o esforço organizacional para a atualização de seus membros perante novas tecnologias.

O segundo fator, carga de trabalho, está relacionado à sobrecarga de atividades ou tarefas exigidas dos funcionários. O suporte material aborda a percepção dos funcionários quanto ao apoio material e à adequação dos recursos oferecidos para desenvolverem suas atividades. $\mathrm{O}$ fator de ascensão, promoção e salários aborda ações organizacionais referentes à retribuição financeira, oportunidades de promoção e ascensão funcional.

Segundo Paschoal, Torres e Porto (2010), o suporte organizacional refere-se a características e a condições de trabalho que podem influenciar diretamente as emoções vivenciadas pelo trabalhador. Por exemplo, ações relacionadas à carga de trabalho podem suscitar experiências afetivas durante o dia a dia organizacional. A má distribuição de tarefas e o excesso quantitativo de atividades poderiam provocar nos trabalhadores emoções negativas, como irritação, raiva e ansiedade.

As ações organizacionais relacionadas à gestão do desempenho e à ascensão profissional, por sua vez, estão associadas a práticas que podem favorecer a experiência de expressão e realização pessoal, como o estímulo e a valorização de novas ideias e oportunidades de promoção. Além disso, é importante destacar que o suporte organizacional envolve a ideia de retribuição organizacional, que pode favorecer as emoções positivas no trabalho e a percepção do indivíduo de que está avançando em suas metas de vida. 
Em pesquisa de campo conduzida em uma organização pública federal, Paschoal et al. (2010) testaram o impacto do suporte organizacional sobre o bem-estar no trabalho. Assim como no presente estudo, a conceituação e a operacionalização do bem-estar consideraram o afeto positivo, o afeto negativo e a realização no trabalho. Os resultados indicaram que a gestão do desempenho e a carga de trabalho consistem em importantes antecedentes do bem-estar. Os fatores ascensão, promoção e salários e suporte material, por sua vez, apresentaram baixa correlação com o bem-estar do trabalhador e não compuseram o modelo preditivo. Maior suporte voltado à progressão na carreira, a salários adequados e a condições materiais não influenciou o afeto ou a realização no trabalho.

Segundo Warr (2007), certas características de trabalho, como ambiente físico, salários e desenvolvimento na carreira, têm impacto no bem-estar quando são percebidas negativamente ou em um nível muito baixo. Na medida em que essas condições começam a se tornar adequadas, seu impacto no bem-estar deixa de ser significativo. Os resultados encontrados por Paschoal et al. (2010) revelaram que o suporte material foi a dimensão de suporte percebida mais positivamente pelos respondentes da organização investigada. Em relação ao suporte para ascensão, promoção e salários, os autores enfatizam que, nas organizações públicas, há estabilidade, cargos e carreiras bem definidos, e mudanças na ascensão estabelecidas a partir do tempo de serviço. Tal estabilidade e fluxo previsível de ações organizacionais podem minimizar o efeito do suporte para ascensão, promoção e salários sobre o afeto e a realização do trabalhador.

Quando são abordadas práticas de gestão de pessoas nas organizações públicas, é necessário considerar uma realidade diferente daquela observada nas organizações privadas (Bergue, 2010). Estas possuem um cenário mais instável e de menor previsibilidade. Especialmente no que diz respeito a ações relacionadas ao crescimento profissional, salários e promoções, as organizações privadas lidam com maior variedade de condições e oportunidades (Bergue, 2010).

Em um contexto de maior instabilidade e competição entre os trabalhadores, as percepções sobre a qualidade do suporte recebido para ascensão e salários podem influenciar diretamente a avaliação do trabalhador sobre sua realização pessoal e as emoções experimentadas diariamente na organização (Paschoal et al., 2010; Warr, 2007). O presente estudo testou a relação da percepção de suporte para ascensão, promoção e salários com afeto positivo, afeto negativo e realização no trabalho em empresas privadas.

\section{Estilos de liderança}

Segundo Lapierre (1989), as atitudes e ações dos líderes de uma empresa exercem importante influência nas percepções e nos comportamentos dos funcionários. Pesquisadores da área do comportamento organizacional destacam que os líderes ou gerentes compõem o grupo de pessoas com maiores níveis de responsabilidade e remuneração em uma organização e influenciam as estratégias e resultados apresentados pelos diferentes grupos (Bergamini, 1994). Dada a relevância que líderes e gestores assumem na compreensão de diversos fenômenos do contexto das organizações, a relação entre seus comportamentos e o bem-estar do trabalhador figura entre questões que precisam ser exploradas na literatura organizacional.

Os estudos de liderança são muito amplos e variados em relação a definições e dimensões do construto (Davel \& Machado, 2001; Vergara, 2007). De acordo com Bergamini (1994), o ponto de convergência entre os autores reside na interpretação de que o fenômeno somente acontece em grupos e onde há um relacionamento de mão dupla entre os envolvidos, na medida em que o líder tenta influenciar seus liderados e os mesmos respondem a tal influência. Portanto, é importante entender que a liderança não ocorre no isolamento, ou seja, não há líderes sem seguidores (Dorfman, 1996). A influência dos líderes ocorre também de forma indireta, quando fornecem recompensas por bons resultados aos subordinados ou ao estabelecerem um relacionamento justo, elevando o moral e o desempenho do grupo.

Segundo Bass (1990), a liderança é uma interação entre dois ou mais membros de um grupo e, frequentemente, envolve a estruturação ou a reestruturação de uma situação, e as percepções e 
expectativas dos membros. Os líderes são agentes de mudança, pessoas cujos atos afetam outras pessoas mais do que as outras pessoas afetam os atos deles. Contudo, não se pode desconsiderar os aspectos ambientais no contexto da liderança. A força das ações do líder sobre o grupo e sobre os resultados organizacionais pode ser limitada pela tecnologia, normas, requerimentos do trabalho e políticas organizacionais (Katz \& Kahn, 1966).

No presente estudo, o termo líder é utilizado como sinônimo de gestor ou gerente. Para autores do campo do comportamento organizacional, liderança e gerência não são exatamente fenômenos idênticos (Robbins, 2005; Schermerhorn, Hunt, Osborn, \& Uhl-Bien, 2010). Enquanto a gerência envolve elaboração de planos formais, desenvolvimento e acompanhamento de estruturas organizacionais e monitoramento de resultados, a liderança envolve o desenvolvimento de uma visão de futuro e a motivação das pessoas para alcance de metas e superação de obstáculos (Robbins, 2005).

A liderança inclui necessariamente a capacidade de influenciar um grupo para alcance de metas, e isso pode decorrer da posição formal ocupada pelo indivíduo na hierarquia organizacional ou não (Robbins, 2005; Schermerhorn et al., 2010). Uma pessoa pode influenciar as outras simplesmente devido à autoridade formal que possui. Mas para compreender bem a liderança, é preciso considerá-la como um fenômeno de influência e confiança (Dorfman, 1996). Um líder poderia, por exemplo, surgir naturalmente em um grupo. Um gerente, por sua vez, poderia influenciar um grupo devido à sua autoridade formal, mas não inspirar e motivar seus subordinados em direção a uma visão de futuro compartilhada.

Apesar das particularidades de cada termo, autores como Dorfman (1996), Melo (2004) e Torres (1999) destacam que influência e confiança são elementos que devem estar presentes na função gerencial. Em seu clássico trabalho The nature of managerial work sobre os papéis desempenhados por gerentes, Mintzberg (1973) defende que todas as atividades gerenciais envolvem direta ou indiretamente liderança. Obter e disseminar informações sobre a organização e seu contexto, planejar e conduzir mudanças, negociar metas e recursos com subordinados e outros atores organizacionais, desenvolver estratégias de motivação e avaliar resultados do grupo são exemplos de tarefas comuns aos gerentes, e relacionadas ao papel de liderança.

No contexto organizacional, portanto, a liderança é uma das funções que deve ser desempenhada pelos gestores. De acordo com Melo (2004), a diferença entre os termos líder e gerente assume pouca importância, pois nos papéis desempenhados pelos gestores, a influência nos grupos e equipes figura entre os principais pré-requisitos. A descrição de elementos como os estilos de liderança adotados pelo gestor e sua eficácia seria mais importante do que a própria distinção dos termos.

Os gestores possuem estilos diferentes de liderar, com foco em elementos diferentes do trabalho, e podem influenciar os comportamentos e os resultados de seus liderados. Dentre os principais estilos observados nas organizações, três se destacam: estilo de gerenciamento voltado para a tarefa, para o relacionamento e para a situação (Melo, 2004). O primeiro estilo, voltado para a tarefa, caracteriza uma forma de gerenciamento em que o líder está preocupado em estruturar o seu papel e o de seus subordinados, designando os empregados para a realização de tarefas, objetivos e metas organizacionais, definindo padrões de desempenho que possibilitem controle, supervisão e fiscalização do comportamento de seus subordinados. O líder geralmente utiliza a hierarquia, as regras e os métodos estabelecidos pela organização para orientar sua atuação. Esse estilo de liderança costuma ser encontrado em ambientes organizacionais onde predominam a autocracia e a falta de autonomia e de participação dos empregados nas decisões (Melo, 2004).

O segundo estilo refere-se ao gerenciamento voltado para os relacionamentos. Os líderes com esse estilo mantêm relações de trabalho baseadas em confiança mútua e amizade com seus subordinados, enfatizando a busca da satisfação dos membros do grupo. As relações interpessoais orientam a atuação do gestor (Melo, 2004). 
Por fim, o estilo de gerenciamento voltado para a situação indica a compreensão e a atuação da liderança a partir das características do líder, dos seus comportamentos e das condições de realização do trabalho. Consiste na habilidade do líder identificar as mudanças do ambiente e se adaptar a elas, variando o seu comportamento de acordo com a maturidade e a capacidade de execução das tarefas de seus subordinados. As ações de gerenciamento consideram as variáveis moderadoras na relação entre a liderança e a eficácia grupal, ou seja, as variáveis de contexto ou situacionais.

Os três estilos de gerenciamento apresentados representam o modo como a liderança é exercida, formatando as relações entre chefes e subordinados. Quando essas relações são permeadas pelo respeito mútuo, pela valorização da competência e da participação dos empregados nas decisões organizacionais ou, por outro lado, quando as relações são de desconsideração das necessidades e das condições dos empregados, com foco exclusivo na realização das metas organizacionais, criam-se condições diversas, que serão percebidas de formas diferentes e poderão gerar impactos positivos ou negativos no trabalhador (Melo, 2004).

Assim, considerando que os líderes podem influenciar experiências positivas ou negativas de seus subordinados, e que os afetos positivos, negativos e realização compõem o bem-estar no trabalho, é possível esperar alguma relação entre os estilos de gerenciamento e o bem-estar. Little, Simmons e Nelson (2007), por exemplo, verificaram que os afetos positivos dos líderes predizem a saúde dos seus liderados. Outras pesquisas empíricas demonstraram que variáveis como o estilo de gerenciamento autocrático e a despreocupação com necessidades, motivações e expectativas dos subordinados potencializam a experiência de estresse dos trabalhadores (O’Driscoll \& Beehr, 1994; Seltzer \& Numerof, 1988).

No presente estudo, foi testada a relação dos estilos gerenciais voltados para a tarefa, para os relacionamentos e para a situação com o afeto positivo, o afeto negativo e a realização no trabalho. Portanto, foram consideradas no modelo preditivo de bem-estar no trabalho tanto percepções sobre ações e práticas da organização (suporte organizacional), quanto percepções sobre ações dos gestores (estilos gerenciais).

\section{Método}

Este foi um estudo de levantamento, com delineamento correlacional, recorte transversal e abordagem quantitativa. Foi conduzido em duas organizações privadas e utilizou um questionário para coleta de dados. A seguir, são oferecidas informações sobre participantes, medidas que compuseram o questionário, procedimentos e análises estatísticas.

\section{Caracterização das organizações e amostra}

A pesquisa foi realizada em duas empresas privadas do setor de vendas e distribuição de bebidas do Distrito Federal. Além de atuarem no mesmo negócio, as duas empresas possuem porte, faturamento, estrutura e condições de trabalho semelhantes. Ambas solicitaram que não fossem identificadas no estudo e que quaisquer dados que pudessem ser associados a elas também não fossem divulgados.

Como um dos objetivos da pesquisa se referia à relação dos estilos gerenciais com o bem-estar no trabalho, participaram do estudo apenas aqueles funcionários que possuíam um gerente específico e bem definido. Em decorrência dos cargos ocupados, nem todos os funcionários das organizações possuem um gerente específico; existem cargos que possuem um ou mais superiores diretos, descaracterizando, assim, uma figura definida de liderança. Consideradas tais condições, apenas deveriam participar do estudo aqueles funcionários que ocupavam cargos de técnicos, analistas administrativos, vendedores ou supervisores de vendas das organizações estudadas. Funcionários que não possuíam contato diário com a empresa, como repositores de mercadoria e motoristas, mesmo que 
tivessem uma figura definida de liderança, não foram incluídos na pesquisa. De acordo com as próprias organizações, a realidade desse grupo de funcionários difere demasiadamente da realidade dos outros.

Na primeira empresa, do total de 223 funcionários, 151 atendiam às condições anteriormente descritas, ou seja, $67,7 \%$ dos funcionários atendiam aos requisitos descritos no parágrafo anterior. Na segunda empresa, do total de 115 funcionários, 54 atendiam às condições para participação no estudo, ou seja, 56,95\% dos funcionários. O instrumento de pesquisa foi aplicado a todos os 205 funcionários das duas empresas que atendiam às condições. Foram devolvidos 178 questionários respondidos, uma taxa de $86,8 \%$ de questionários devolvidos. Após a exclusão de questionários preenchidos de forma incompleta, 157 questionários válidos foram tabulados e analisados. Assim, do total de funcionários que atendiam aos requisitos para participação, $76,6 \%$ compuseram a amostra de pesquisa.

A amostra ficou composta por 116 funcionários da primeira empresa e 41 da segunda empresa. Quanto aos dados sociodemográficos dos participantes, $84 \%$ dos respondentes eram homens. A média de idade foi de 27 anos ( $d p=6$ anos). O tempo médio de empresa foi de 2,75 anos ( $d p=2,27$ anos). Dentre os respondentes que indicaram sua função ou cargo, 97 eram vendedores, 19 eram supervisores, 14 eram técnicos e 12 tinham cargo de analistas administrativos.

\section{Instrumento}

Para mensurar as variáveis abordadas, foi aplicado um questionário composto pelas seguintes medidas: Escala de Bem-estar no Trabalho (EBET), Escala de Avaliação do Estilo Gerencial (EAEG), conjunto de itens referentes à ascensão, promoção e salários da Escala de Suporte Organizacional Percebido (ESOP) e itens sobre variáveis sociodemográficas.

A Escala de Bem-estar no Trabalho (EBET), validada por Paschoal e Tamayo (2008), é composta por duas partes, uma referente à dimensão afetiva do bem-estar no trabalho e outra referente à realização no trabalho. Dispõe de 30 itens divididos em três fatores: afeto positivo, com nove itens e índice de confiabilidade alpha de Cronbach $(\alpha)$ de 0,91; afeto negativo, com 12 itens e $\alpha$ de 0,89; realização, composto por nove itens e $\alpha$ equivalente a 0,88 . Os itens de afeto no trabalho, como animado, entusiasmado, frustrado e deprimido, devem ser respondidos de acordo com a seguinte instrução: nos últimos seis meses, o meu trabalho tem me deixado. A escala de resposta varia de 1 (nem um pouco) a 5 (extremamente). Os itens de realização, como realizo atividades que expressam minhas capacidades e expresso o que há de melhor em mim, devem ser respondidos de acordo com a seguinte instrução: neste trabalho. A escala de resposta varia de 1 (discordo totalmente) a 5 (concordo totalmente).

A Escala de Avaliação do Estilo Gerencial (EAEG), validada por Melo (2004), contém 19 itens, divididos em três fatores. Um total de seis itens, como valoriza a disciplina e a subordinação, referese ao estilo de gerenciamento voltado à tarefa, $\operatorname{com} \alpha$ de 0,83 ; nove itens, como é atencioso no relacionamento com os subordinados, referem-se ao estilo voltado para o relacionamento, com $\alpha$ de 0,93; quatro itens, como dá maior ou menor liberdade de trabalho ao subordinado, dependendo da sua competência para realizar a tarefa, mensuram um estilo voltado à situação, com $\alpha$ equivalente a 0,85 . Os itens devem ser respondidos de acordo com a instrução: indique o quanto as questões correspondem à maneira como a sua chefia imediata se comporta na organização. A escala de resposta varia de 1 (nunca age assim) a 5 (sempre age assim).

Por fim, foi utilizada a parte sobre percepção de suporte para ascensão, promoção e salários da Escala de Suporte Organizacional Percebido (ESOP), validada por Tamayo, Pinheiro, Tróccoli e Paz (2000). Ao todo, são cinco itens, como nesta organização, as oportunidades de ascensão são compatíveis com as aspirações dos funcionários, que devem ser respondidos de acordo com uma escala que varia de 1 (nunca) a 5 (sempre). O $\alpha$ desse fator da ESOP é de 0,79.

A Tabela 1 resume as informações das medidas utilizadas. 
Tabela 1

Características das Medidas Utilizadas

\begin{tabular}{cccc}
\hline Medidas & Fatores & Número de Itens & Alpha \\
& & & \\
\hline \multirow{2}{*}{ EBET } & Afeto Positivo & 9 & 0,91 \\
& Afeto Negativo & 12 & 0,89 \\
& Realização & 9 & 0,88 \\
\hline \multirow{2}{*}{ EAEG } & Tarefa & 6 & 0,83 \\
& Relacionamento & 9 & 0,93 \\
& Situação & 4 & 0,85 \\
\hline ESOP & Ascensão, Promoção e Salários & 5 & 0,79 \\
\hline
\end{tabular}

\section{Procedimentos de coleta e análise de dados}

Um dos pesquisadores, que atuava como estagiário em uma das organizações participantes, entrou em contato com o gestor da área de Gestão de Pessoas para apresentar a pesquisa e convidar a organização para participar. O projeto de pesquisa foi analisado e aprovado pelos representantes da área. Em seguida, foram negociadas a coleta de dados e a divulgação da pesquisa aos funcionários que preenchiam os requisitos para participação. Os próprios requisitos para inclusão dos funcionários também foram discutidos, pois era necessário saber que tipo de funcionário atenderia às demandas da pesquisa.

O gestor da área de gestão de pessoas enviou um correio eletrônico aos funcionários, informando o objetivo da pesquisa, o caráter acadêmico desta e a não vinculação dos resultados, ações e decisões organizacionais, além das questões relacionadas ao sigilo e à voluntariedade da participação. Também informou que receberiam a visita do pesquisador em seu local de trabalho.

O pesquisador, então, agendou com o gestor horários em que poderia transitar pela organização e abordar os funcionários. Os questionários foram aplicados coletivamente no local de trabalho durante o horário de expediente. Como muitos participantes sentavam-se próximos uns aos outros, separados apenas por baias ou mesas diferentes, o pesquisador aproximava-se desses funcionários e fazia o convite para que ouvissem a apresentação do trabalho, incluindo objetivos, caráter acadêmico, sigilo e não obrigatoriedade de participação. Como os funcionários já haviam sido informados da pesquisa, a maioria aceitava ouvir a apresentação e participar. Aqueles que informavam que não poderiam ouvir a apresentação ou responder naquele momento eram abordados em outro horário e outra visita.

Os questionários eram distribuídos e os participantes respondiam em seus locais e mesas de trabalho. O pesquisador esperava próximo aos respondentes e ficava à disposição para esclarecimento de dúvidas. Imediatamente após o preenchimento das respostas, que levava entre 10 e 15 minutos, os participantes devolviam o questionário para o pesquisador, que o colocava em um envelope, o qual lacrava.

O mesmo procedimento foi seguido na segunda organização participante. Nenhum dos pesquisadores possuía qualquer vínculo com a segunda organização ou com seus gestores, mas foi feito contato telefônico com o gestor da área de Gestão de Pessoas, que recebeu os pesquisadores e submeteu o projeto à avaliação dos representantes da área. Após a aprovação, foram seguidos os mesmos passos adotados na primeira organização.

Para que todos os funcionários que se encaixavam nos requisitos para participação da pesquisa pudessem ser acessados, foram realizadas várias visitas do pesquisador aos locais de trabalho dos 
participantes, em horários agendados com o gestor de pessoas de cada organização. A coleta teve duração aproximada de quatro semanas e todos os potenciais participantes foram acessados e responderam ao questionário. Conforme destacada na seção sobre características das organizações e amostra, $76,6 \%$ dos questionários devolvidos estavam completos e foram considerados na pesquisa.

A variável dependente do estudo foi o bem-estar no trabalho, e está composta por três fatores: afeto positivo, afeto negativo e realização. As variáveis independentes foram os três fatores de estilos de gerenciamento e a percepção de suporte para ascensão, promoção e salários. Inicialmente, as variáveis foram descritas por meio de medidas de centralidade e dispersão. Posteriormente, as associações entre as variáveis foram exploradas por meio de correlação bivariada de Pearson. Em função dos resultados encontrados, foram conduzidas a rotação e a extração dos fatores para os itens de estilos de gerenciamento por meio do método PAF (Principal Axis Factoring), com rotação oblíqua. Por fim, foram conduzidas regressões múltiplas padrões.

\section{Resultados}

Como a amostra foi composta por participantes de empresas diferentes, foram comparados os resultados dos dois grupos por meio de análise de variância. Não foram encontradas diferenças estatísticas significativas entre os participantes das duas empresas em relação a qualquer variável. A Tabela 2 indica os valores das médias, desvios padrões, medianas, mínimos e máximos para os dois grupos.

Conforme mencionado na descrição das organizações e participantes, as empresas abordadas eram semelhantes em relação ao negócio, faturamento, estrutura e condições de trabalho. A experiência de bem-estar no trabalho e as percepções sobre suporte e estilos gerenciais observadas nas duas empresas foram as mesmas.

Tabela 2

Resultados Descritivos para os Dois Grupos de Participantes

\begin{tabular}{|c|c|c|c|c|c|c|c|c|c|c|}
\hline \multirow[b]{2}{*}{ Variável } & \multicolumn{5}{|c|}{$\begin{array}{c}\text { Empresa } 1 \\
(\mathrm{~N}=116)\end{array}$} & \multicolumn{5}{|c|}{$\begin{array}{c}\text { Empresa } 2 \\
(\mathrm{~N}=41)\end{array}$} \\
\hline & M & DP & $\mathrm{Md}$ & Min & Máx & M & $\mathrm{DP}$ & Md & Min & Máx \\
\hline 1. Afeto Positivo & 3,02 & 0,90 & 3,00 & 1,0 & 5,0 & 3,14 & 0,75 & 3,01 & 1,4 & 4,5 \\
\hline 2. Afeto Negativo & 2,40 & 0,80 & 2,20 & 1,0 & 4,6 & 2,10 & 0,60 & 1,90 & 1,0 & 3,5 \\
\hline 3. Realização & 3,75 & 0,72 & 3,85 & 1,7 & 5,0 & 3,90 & 0,76 & 3,90 & 1,0 & 4,9 \\
\hline 4. Suporte Ascensão... & 2,95 & 0,85 & 3,00 & 1,0 & 4,8 & 3,10 & 0,80 & 3,00 & 1,8 & 4,8 \\
\hline 5. Estilo Tarefa & 3,95 & 0,70 & 4,00 & 1,0 & 5,0 & 4,20 & 0,60 & 4,50 & 2,5 & 5,0 \\
\hline 6. Estilo Situação & 4,00 & 0,80 & 4,00 & 1,0 & 5,0 & 4,20 & 0,70 & 4,20 & 2,0 & 5,0 \\
\hline 7. Estilo Relacionamento & 3,90 & 0,90 & 4,10 & 1,0 & 5,0 & 4,10 & 0,60 & 4,20 & 2,30 & 5,0 \\
\hline
\end{tabular}

Considerando a ausência de diferenças significativas entre os dois grupos para todas as variáveis, as análises posteriores foram conduzidas considerando os respondentes como um mesmo grupo. Os resultados das médias e desvios padrões gerais e das correlações entre as variáveis encontram-se na Tabela 3. 
Tabela 3

\section{Correlações entre Variáveis do Estudo}

\begin{tabular}{lccccccccc}
\hline Variável & M & DP & 1 & 2 & 3 & 4 & 5 & 6 & 7 \\
\hline 1. Afeto Positivo & 3,06 & 0,89 & 1 & & & & & & \\
2. Afeto Negativo & 2,36 & 0,79 & $-0,60^{* *}$ & 1 & & & & & \\
3. Realização & 3,79 & 0,74 & $0,55^{* *}$ & $-0,31^{*}$ & 1 & & & & \\
4. Suporte Ascensão... & 2,98 & 0,86 & $0,52^{* *}$ & $-0,36^{*}$ & $0,49^{* *}$ & 1 & & & \\
5. Líder Tarefa & 4,04 & 0,73 & $0,34^{* *}$ & $-0,30^{*}$ & $0,31^{* *}$ & $0,35^{* *}$ & 1 & & \\
6. Líder Situação & 4,03 & 0,82 & $0,33^{* *}$ & $-0,30^{*}$ & $0,32^{* *}$ & $0,38^{* *}$ & $0,74^{* *}$ & 1 & \\
7. Líder Relacionamento & 3,99 & 0,82 & $0,41^{* *}$ & $-0,37^{*}$ & $0,34^{* *}$ & $0,40^{* *}$ & $0,78^{* *}$ & $0,87^{* *}$ & 1 \\
\hline
\end{tabular}

Nota. $* \mathrm{p}<0,05 * * \mathrm{p}<0,01$.

Todas as variáveis independentes apresentaram correlações significativas com os fatores de bem-estar. Com afeto positivo e realização, as associações das variáveis de suporte e estilos de liderança foram positivas; com afeto negativo, as associações foram negativas. As associações mais fortes foram observadas entre a percepção de suporte para ascensão, promoção e salários e os fatores afeto positivo $(0,52)$ e realização $(0,49)$.

Sobre os estilos de gerenciamento, as correlações entre os três fatores foram altas, positivas e significativas. No estudo de Melo (2004), o autor identificou três fatores distintos para os estilos de gerenciamento. Porém, no presente estudo, os três estilos pareceram não se diferenciar na percepção dos respondentes. Devido à alta correlação entre os fatores de estilos gerenciais, foi conduzida uma análise fatorial exploratória para testar a distribuição dos itens nos fatores propostos e verificar se alguns destes poderiam ser agrupados. Não foi objetivo do presente estudo validar o instrumento de estilos de gerenciamento, que já foi testado por Melo (2004). Apenas foi verificada a estrutura dos itens mediante as altas correlações encontradas. Para tal fim, foi utilizado o método PAF com rotação oblíqua.

Os resultados não sustentaram a proposição de agrupar os itens em torno de três fatores. A análise do screeplot indicou que os itens poderiam ser agrupados em torno de duas dimensões. Após a rotação e a extração dos dois fatores, a estrutura encontrada apresentou sentido teórico: o primeiro ficou constituído pelos itens de estilo voltado ao relacionamento e estilo voltado à situação; o segundo fator contemplou os itens de estilo voltado à tarefa. A fim de assegurar que cada item representava o construto subjacente ao fator, foi estipulada uma carga mínima de 0,30 para aceitar o item.

O primeiro fator ficou composto por 12 itens e apresentou coeficiente $\alpha$ de 0,84 . O segundo ficou composto por quatro itens e apresentou um coeficiente $\alpha$ de 0,79 . Três itens foram excluídos por apresentarem carga nos dois fatores e, portanto, não se mostrarem como operacionalização adequada de um ou outro construto. Por exemplo, o item é rígido no cumprimento de prazos, que foi excluído, pode não ser considerado uma descrição exclusiva de um líder com estilo voltado à tarefa. Mesmo com a retirada, a consistência dos fatores foi satisfatória.

O agrupamento dos itens referentes aos dois estilos de gerenciamento (relacionamento e situação) faz sentido do ponto de vista da proximidade de seus conteúdos, os quais não são excludentes. Tanto o estilo relacionamento quanto o estilo situação apresentam maneiras de gerenciar que privilegiam ações voltadas para as pessoas, para as condições que podem facilitar a realização do trabalho do subordinado e o alcance dos objetivos pessoais e organizacionais. São, portanto, maneiras de gerenciar que podem se complementar. 
Quanto às correlações dos novos fatores de estilo de gerenciamento com o bem-estar, as associações foram todas significativas $(\mathrm{p}<0,01)$. O estilo voltado para relacionamento/situação correlacionou-se negativamente com afeto negativo $(-0,36)$ e positivamente com afeto positivo $(0,39)$ e realização $(0,35)$. Já o estilo voltado para tarefa correlacionou-se negativamente com afeto negativo $(-0,24)$ e positivamente com afeto positivo $(0,30)$ e realização $(0,26)$.

Da análise das correlações entre variáveis independentes e dependentes, os resultados demonstram que existem associações significativas entre suporte, estilos de liderança e bem-estar. Partiu-se, assim, para a condução de regressões do tipo padrão para testar a força de cada variável em um modelo preditivo das dimensões do bem-estar no trabalho. Para a condução das regressões, foram considerados os dois fatores de estilos de gerenciamento.

A Tabela 4 apresenta os resultados encontrados para cada variável dependente: afeto positivo, afeto negativo e realização. São indicados os coeficientes de regressão padronizados $(\beta)$, o poder explicativo de cada modelo $\left(\mathrm{R}^{2}\right)$ e sua significância. Quanto à regressão do suporte para ascensão, promoção e salários, e estilos de gerenciamento sobre o afeto positivo, o modelo encontrado foi significativo $\left(\mathrm{F}_{3,153}=23,338, \mathrm{p}<0,001\right)$ e composto pelas variáveis suporte $(\beta=0,43)$ e estilo voltado para relacionamento/situação $(\beta=0,20)$.

Juntos, suporte para ascensão, promoção e salários, e estilo de gerenciamento voltado para relacionamento/situação, explicaram $30 \%$ do afeto positivo no trabalho. Suporte, por sua vez, foi a variável que apresentou maior poder explicativo, corroborando os resultados encontrados nas correlações. A relação entre as variáveis independentes apontadas e o afeto positivo foi positiva, indicando que quanto mais positiva é a percepção de suporte e mais presente é o estilo relacionamento/situação, mais afeto positivo é vivenciado no trabalho.

Tabela 4

Resultados da Regressão Múltipla Padrão para Cada Variável Dependente

\begin{tabular}{lccc}
\hline & Afeto Positivo & Afeto Negativo & Realização \\
\cline { 2 - 4 } \multicolumn{1}{c}{ Variáveis Independentes } & $\beta$ & $\beta$ & $\beta$ \\
\hline Suporte Ascensão... & $0,43^{* *}$ & $-0,26^{* *}$ & $0,41^{* *}$ \\
Estilo Relacionamento/Situação & $0,20^{*}$ & $-0,25^{*}$ & 0,13 \\
Estilo Tarefa & 0,03 & $-0,01$ & 0,07 \\
\hline $\mathrm{R}^{2}$ ajustado & 0,30 & 0,18 & 0,26 \\
\hline
\end{tabular}

Nota. $* \mathrm{p}<0,05 ; * * \mathrm{p}<0,001$.

Quanto à regressão do suporte para ascensão, promoção e salários, e estilos de gerenciamento sobre $\mathrm{o}$ afeto negativo, o modelo encontrado foi significativo $\left(\mathrm{F}_{3,153}=12,175, \mathrm{p}<0,001\right)$ e composto pelas variáveis suporte $(\beta=-0,26)$ e estilo voltado para relacionamento/situação $(\beta=-0,25)$. Juntas, elas explicaram $20 \%$ do afeto negativo no trabalho. Assim, como no caso do afeto positivo, suporte foi a variável que apresentou maior poder explicativo. A relação entre as variáveis independentes apontadas e o afeto negativo foi negativa, indicando que quanto mais positiva é a percepção de suporte e mais presente é o estilo relacionamento/situação, menos afeto negativo é vivenciado no trabalho.

Por fim, foi conduzida a regressão para o fator de realização. O modelo encontrado foi significativo $\left(\mathrm{F}_{3,153}=10,044, \mathrm{p}<0,001\right)$ e composto pela variável suporte para ascensão, promoção e salários $(\beta=0,41)$, que explicou, sozinha, $27 \%$ da realização. A relação entre as variáveis foi positiva, indicando que, quanto mais positiva é a percepção de suporte, mais realização pessoal é vivenciada pelo trabalhador. 


\section{Discussão}

Os resultados referentes às análises descritivas das variáveis investigadas indicam que os participantes vivenciam moderadas experiências positivas no trabalho e, portanto, moderado bemestar, visto que os valores para afeto positivo e realização encontram-se ligeiramente acima do ponto médio da escala de resposta. Apesar disso, a média para afeto negativo também foi muito próxima do ponto médio da escala, indicando que esta também é uma experiência significativa dos trabalhadores.

Os resultados sustentam proposições e achados de diversos autores da área, segundo os quais, afeto positivo e negativo não são exatamente excludentes e podem ser vivenciados concomitantemente (Ryan \& Deci, 2001; Warr, 2007; Waterman, Schwartz, \& Conti, 2008). A presença de emoções e humores positivos não significa a ausência de emoções e humores negativos. Para os participantes desta pesquisa, o trabalho na organização está permeado tanto de afeto positivo quanto negativo.

Sobre a percepção de suporte para ascensão, promoção e salários, a pontuação média dos respondentes encontra-se próxima do ponto médio da escala de resposta, o que sugere uma percepção razoavelmente positiva deste fator. A avaliação do respondente para a ocorrência de ações organizacionais que expressam suporte poderia variar de nunca a sempre. A pontuação muito próxima ao ponto médio da escala indica que às vezes são percebidas ações dessa natureza. O ideal é que as ações fossem percebidas como frequentes. Segundo Tamayo et al.(2000), quanto mais frequentes as ações, mais positiva é a avaliação do trabalhador.

A percepção deste tipo de suporte poderia ser melhorada a partir de ações das organizações. De acordo com Eisenberger et al. (1986), a percepção de suporte reflete a qualidade do tratamento que o trabalhador recebe da organização, ou seja, quanto maior a percepção de suporte, mais os funcionários estão dispostos a retribuir à organização com seus esforços e dedicação. Analisando a percepção dos participantes, a retribuição do trabalhador pode acompanhar o índice mediano encontrado na pesquisa, sugerindo, assim, a importância de investimentos em oportunidades de crescimento pessoal e profissional.

Em relação aos estilos de gerenciamento, não foram identificados os três fatores propostos por Melo (2004). Pelo menos na percepção da amostra investigada, o construto se mostrou complexo e os três estilos não se diferenciaram. A extração de fatores conduzida sugeriu que os itens poderiam ser organizados em torno de dois fatores: estilo voltado para relacionamento/situação e estilo voltado para tarefa.

É importante notar que esses achados são compatíveis com resultados dos primeiros estudos sobre liderança, quando eram enfatizados apenas dois tipos de estilo de gerenciamento (Dorfman, 1996; Torres, 1999). Basicamente, os estilos poderiam se voltar para empregado e relações ou para produção, papéis e metas. A organização dos itens em torno de duas dimensões demonstra a força desses dois conteúdos (relação e tarefa) na formação do significado e da compreensão do fenômeno da liderança, embora seja preciso alertar para a não inclusão das variáveis situacionais em nenhum desses dois estilos de gerenciamento.

As médias elevadas observadas para todos os estilos gerenciais e as altas correlações entre eles podem indicar a presença dos três tipos de líderes nas organizações, ou mesmo que os líderes emitem comportamentos de todos os tipos, dependendo da situação de trabalho apresentada. Segundo a percepção dos respondentes, não existe um estilo de gerenciamento exclusivo predominante no ambiente das duas organizações pesquisadas. Poder-se-ia questionar até mesmo se não existe um único estilo de liderança composto pela combinação e mistura de comportamentos de tarefa e relacionamento/situação.

A maneira de gerenciar parece se adequar à situação e às necessidades que se apresentam (Bass, 1990; Torres, 1999). Ora os gestores expressam uma maneira de gerenciar voltada prioritariamente para as tarefas, possivelmente nos momentos em que há urgência em apresentar resultados em curto 
prazo, ora os gestores envolvem e consideram os interesses, necessidade e dificuldades das pessoas em seu gerenciamento. Em certos momentos, outros elementos ainda poderiam ser equacionados pelos gestores em sua maneira de gerenciar e tomar decisões. Os resultados sugerem que categorias muito claras, precisas e diferenciadas sobre os estilos de gerenciamento parecem não ser facilmente percebidas no complexo contexto organizacional.

Pode-se cogitar a possibilidade de tais resultados terem sofrido influência de algum desejo social por parte dos respondentes. Apesar dos cuidados com o sigilo das respostas, com as instruções e a aplicação dos questionários, os participantes podem ter respondido o que acreditavam que era esperado, seja por receio de que o gerente pudesse ter acesso a suas respostas ou de que estas pudessem afetar positiva ou negativamente os seus gestores. Por outro lado, é importante enfatizar que o conteúdo dos fatores de estilos de gerenciamento e sua operacionalização devem ser alvo de novas pesquisas. Em estudo anterior, Meleiro (2005) utilizou o instrumento de Melo (2004) e encontrou as mesmas altas correlações verificadas na presente pesquisa.

Quanto às relações dos dois estilos de liderança com o bem-estar, os resultados das correlações indicaram que tanto o estilo voltado para relacionamento/situação quanto o estilo voltado para tarefa apresentam associações significativas e positivas com afeto positivo e realização, e associações significativas e negativas com afeto negativo. Tais resultados sugerem que o simples gerenciamento do trabalho e a presença do líder no dia a dia organizacional, independente do estilo adotado, têm associação com o bem-estar no trabalho.

Quando considerados os resultados da regressão, porém, apenas o estilo voltado para relacionamento/situação compôs o modelo preditivo de afeto positivo e afeto negativo. Quando os gestores se comportam considerando as necessidades dos subordinados, a qualidade dos relacionamentos e as condições que podem facilitar a realização do trabalho, emoções e humores positivos, como prazer, entusiasmo e tranquilidade, tendem a aumentar, e emoções e humores negativos, como ansiedade, raiva e depressão, tendem a diminuir.

Nenhum estilo gerencial compôs o modelo preditivo de realização. As ações e comportamentos dos líderes se relacionaram com as reações afetivas do trabalhador em sua rotina de trabalho, mas não se relacionaram com sua percepção de que metas de vida estão sendo alcançadas, e os potenciais individuais estão sendo expressos e desenvolvidos. De acordo com Gray e Watson (2001), as emoções são reações que promovem a sobrevivência do organismo e facilitam respostas adaptativas às mudanças do ambiente. As emoções e os humores refletem a posição do organismo na interação com o meio.

Os comportamentos dos líderes criam condições e demandas de trabalho às quais os subordinados precisam se adaptar diariamente. As experiências afetivas são as primeiras e principais reações do trabalhador a tais demandas. Estudos anteriores demonstraram que comportamentos do líder influenciam o estresse do trabalhador, considerado um fenômeno essencialmente afetivo (O’Driscoll \& Beehr, 1994; Seltzer \& Numerof, 1988). A realização, por sua vez, parece não depender de ações específicas dos líderes nem de demandas geradas por seu comportamento, mas de condições de trabalho estabelecidas pela organização. Autores como Warr (2007) e Waterman et al. (2008) já salientaram que a experiência de realização pessoal é mais complexa que os afetos, e está relacionada a uma combinação de variáveis organizacionais e pessoais, podendo-se concluir, portanto, que está além do comportamento do líder.

Dentre as variáveis independentes, o principal preditor de todos os fatores de bem-estar foi o suporte organizacional para ascensão, promoção e salários. Tais resultados diferem daqueles encontrados por Paschoal et al. (2010), que revelaram que o suporte para ascensão, promoção e salários tem baixa correlação com os fatores de bem-estar no trabalho e não predizem sua ocorrência.

$\mathrm{Na}$ presente pesquisa, o suporte para ascensão, promoção e salários apresentou-se como um fator importante para todas as dimensões do bem-estar. Indica que as organizações deveriam se preocupar em desenvolver ações que melhorem a opinião dos trabalhadores acerca de retribuições 
financeiras e benefícios, perspectivas de promoção salarial ou funcional, e ascensão e desenvolvimento de carreira.

Enquanto os servidores das organizações públicas contam com estabilidade, e a progressão na carreira é baseada especialmente no tempo de trabalho, as promoções e a progressão nas empresas privadas são menos previsíveis, podendo demandar maior esforço e competição interpessoal por parte dos trabalhadores (Bergue, 2010). O desenvolvimento profissional mais vinculado à competição e, possivelmente, a uma maior escassez de oportunidades, torna o trabalhador mais sensível a esse tipo de prática organizacional.

Em sua revisão sobre antecedentes do bem-estar no trabalho, Warr (2007) destaca o modelo vitamínico sobre impacto de variáveis contextuais no bem-estar. O modelo assume que o bem-estar é influenciado pelo ambiente de maneira análoga ao efeito das vitaminas sobre a saúde física. A presença de algumas vitaminas no organismo é importante para a saúde, mas, depois de atingir certo nível, não há mais benefício derivado de quantidades adicionais. Características como dinheiro, segurança física e posição social teriam um tipo de impacto semelhante no bem-estar. Se insatisfatórias, influenciaram negativamente o bem-estar. Depois de atingir certo nível, o aumento dessas características não aumentaria o bem-estar.

A analogia entre necessidades de vitaminas e demandas do meio apresenta alguns problemas. Por exemplo, um enfoque derivado de processos químicos não pode ser transferido a um contexto de relações sociais como o de trabalho. As necessidades humanas em relação ao seu meio são também de caráter simbólico, composta por aspectos cognitivos, motivacionais e afetivos.

Apesar das fragilidades, Warr (2007) destaca como vantagem do modelo vitamínico a ênfase na questão dos diferentes efeitos que o meio pode ter sobre o bem-estar. O presente estudo não testou relações curvilíneas entre suporte e bem-estar, mas os resultados oferecem alguma sustentação à proposta de Warr (2007). Se o suporte para ascensão, promoção e salários não é totalmente satisfatório, é possível que quanto melhor a avaliação, maior o bem-estar. As organizações públicas, por sua vez, contam com estabilidade e previsibilidade quanto a aspectos de ascensão, promoção e salários (Bergue, 2010) e, portanto, os resultados do estudo de Paschoal et al. (2010) foram distintos.

Há que se considerar que, além da diferença entre as organizações do estudo de Paschoal et al. (2010) e do estudo atual, a amostra foi composta por categorias profissionais diferentes. Naquele, participaram trabalhadores que exerciam funções administrativas e, neste, participaram vendedores. $\mathrm{O}$ tipo de função desempenhada pelo trabalhador poderia ser uma variável interveniente nas relações entre variável independente e dependente. Para uma comparação sistemática dos resultados, seria desejável a replicação da pesquisa com as mesmas categorias profissionais.

De forma geral, os resultados encontrados nesta pesquisa indicam que reações emocionais e avaliações sobre realização pessoal podem receber impacto direto do tipo de suporte em questão. Além disso, quando comparadas com ações dos gestores, ações organizacionais que apoiam carreira e remuneração do trabalhador exercem um papel mais importante para explicação do bem-estar. Eisenberger et al. (1986) sugere a importância de ações organizacionais para comportamentos diversos dos trabalhadores, pois o indivíduo geralmente atribui práticas da sua rotina de trabalho à organização e não a agentes específicos.

É significativo destacar a importância de variáveis organizacionais, ainda que sejam avaliadas por meio de percepções dos trabalhadores, para explicação do bem-estar. A literatura tem constantemente demonstrado o poder preditivo de tais variáveis (Dessen \& Paz, 2010; Paz et al., 2009; Silva \& Ferreira, 2011; Wilson et al., 2004). Os resultados presentes vão ao encontro das pesquisas anteriores.

Apesar das correlações entre variáveis independentes e dependentes deste estudo não serem elevadas, e os modelos encontrados não explicarem mais que $30 \%$ da variância de cada dimensão do bem-estar, fenômenos do campo do comportamento organizacional são multideterminados. Correlações e modelos preditivos resultantes de estudos empíricos da área geralmente apresentam 
valores considerados fracos ou moderados (Abbad \& Torres, 2002; Warr, 2007). Barsky e Kaplan (2007), por exemplo, verificaram em sua meta-análise que a associação da justiça organizacional com o afeto no trabalho indicaram valores de correlação entre 0,20 a 0,31 .

Finalmente, este estudo vai ao encontro de propostas teóricas (Ryan \& Deci, 2001; Warr, 2007; Waterman et al., 2008) e estudos empíricos (Ferreira et al., 2008; Paschoal \& Tamayo, 2008) que defendem que o ambiente organizacional é um contexto permeado por emoções, humores e experiências cognitivas de realização pessoal. Ainda que a empresa tenha seus objetivos e metas, que devem ser alcançados, os trabalhadores reagem emocionalmente às práticas e ações organizacionais, e desenvolvem expectativas e avaliações quanto à possibilidade de expressar seus potenciais e avançar em direção a metas pessoais. Assim, as práticas organizacionais devem oferecer condições de trabalho adequadas e permitir que o trabalhador encontre suporte para seu desenvolvimento pessoal e profissional (Warr, 2007; Waterman et al., 2008).

\section{Conclusões}

O presente estudo tratou de investigar a relação de variáveis organizacionais e laborais percebidas com o bem-estar no trabalho. Apesar do constante interesse dos pesquisadores organizacionais em compreender os antecedentes do bem-estar do trabalhador, muitas lacunas ainda permanecem na literatura científica, seja em relação à definição e à operacionalização do construto ou em relação a questões metodológicas e de generalização de resultados.

Uma primeira contribuição deste estudo refere-se ao próprio construto e à operacionalização do bem-estar no trabalho. Aquele foi conceituado e mensurado a partir de uma dimensão afetiva (emoções e humores) e de uma dimensão cognitiva (realização pessoal no trabalho), abordando dois elementos considerados centrais para a compreensão do fenômeno e, muitas vezes, não incluídos nas pesquisas.

Além disso, foram testadas relações com variáveis que ainda não foram sistematicamente associadas com as várias dimensões do bem-estar no trabalho. Apesar da atenção que variáveis como estilos de liderança vêm recebendo no campo do comportamento organizacional e de gestão de pessoas, existe uma grande variedade de modelos sobre o fenômeno, o que dificulta a sistematização dos achados. Esta pesquisa utilizou um modelo e um instrumento de estilos de liderança testado em organizações brasileiras e investigou as relações dos fatores propostos com os elementos afetivos e de realização do bem-estar. A revisão de literatura indicou que não haviam sido realizadas pesquisas com tais variáveis, da forma como foram conceituadas e operacionalizadas.

Foi possível também investigar a relação do bem-estar com dois tipos diferentes de variáveis relacionadas ao trabalho: estilos de liderança, que se referem a ações e a comportamentos de atores específicos da organização, e suporte organizacional, que aborda a avaliação do sujeito sobre a organização como um todo e suas práticas.

Os resultados encontrados indicaram que, nas empresas privadas investigadas, o suporte organizacional para ascensão, promoção e salários consiste em ações organizacionais importantes para a explicação do afeto e da realização do trabalhador. Além disso, quando comparado aos estilos de gerenciamento, o suporte apresenta um poder preditivo maior. A literatura organizacional tem atribuído uma grande responsabilidade aos líderes sobre resultados e comportamentos individuais e grupais da organização. Os resultados desta pesquisa contribuem para levantar questões sobre a importância das ações isoladas dos líderes e sobre como ou em quais condições seu impacto no trabalhador é maior.

Em termos práticos, os resultados indicam para os gestores a necessidade da implantação de práticas organizacionais que permitam uma retribuição compatível com os esforços individuais. Ações específicas dos líderes em seus grupos e áreas podem influenciar as respostas afetivas dos 
trabalhadores, mas para possibilitar a expressão e a realização pessoal no trabalho, parecem ser necessárias ações de maior amplitude, que representem práticas da organização como um todo (Eisenberger et al., 1986; Paschoal et al., 2010; Warr, 2007).

Estratégias e ações para valorização e desenvolvimento dos ativos humanos devem, primeiramente, ser avaliadas como justas pelos trabalhadores. No que diz respeito ao suporte para promoção, ascensão e salários, nem sempre a empresa pode alterar salários e aumentar recompensas financeiras. Apesar disso, pode-se desenvolver políticas e práticas relacionadas a caminhos alternativos para desenvolvimento da carreira, que não dependam exclusivamente de promoção para cargos de chefia, como enriquecimento de atividades laborais, atribuição ao trabalhador de maior autonomia e controle sobre suas próprias tarefas e outras formas de reconhecimento valorizadas pelos integrantes da organização.

Apesar das contribuições e das conclusões que podem ser extraídas do estudo, limitações alertam para que os resultados sejam considerados com cautela. Certamente, os resultados encontrados não podem ser generalizados para outras empresas e contextos laborais, mas levantam questões que deverão ser exploradas em pesquisas futuras. $\mathrm{O}$ aprofundamento das dimensões dos estilos gerenciais e as relações entre eles é uma delas. O campo da liderança é repleto de propostas teóricas. Os achados deste estudo sugerem que o modelo e o instrumento desenvolvidos no Brasil merecem a atenção dos pesquisadores. Uma cuidadosa análise teórica dos fatores e uma posterior análise da operacionalização dos itens deverão ser conduzidas.

Em relação a antecedentes do bem-estar no trabalho, o efeito de outras variáveis organizacionais e laborais percebidas deve ser investigado. Pesquisas em ambiente organizacional com rotinas aceleradas e alta cobrança de resultados demandam cuidados especiais na negociação e no planejamento para coleta de dados. Os questionários, por exemplo, precisam ser resumidos e nem sempre é possível incluir medidas de todas as variáveis que, teoricamente, poderiam explicar o bemestar. Neste estudo, optou-se pela inclusão de um dos fatores de suporte organizacional e de três estilos de liderança.

Para a coleta de dados, foi necessário compatibilizar demandas dos pesquisadores e das organizações. Os dados foram coletados no próprio ambiente de trabalho dos respondentes e, em uma das organizações, um dos pesquisadores exercia atividades de estágio. Mesmo com o cuidado para explicação do caráter acadêmico da pesquisa e para preservação do sigilo dos respondentes, é possível que tenha havido impacto de alguma desejabilidade social, aspecto já levantado na discussão sobre resultados referentes aos estilos de liderança.

Outra questão que merece destaque refere-se ao nível de análise das variáveis. O bem-estar no trabalho refere-se a uma experiência subjetiva do trabalhador; são experiências internas do indivíduo e nenhuma condição externa pode definir o construto. Assim, o bem-estar no trabalho deve ser considerado no nível individual de análise. O suporte organizacional, por sua vez, consiste em ações e práticas da organização e, assim, poderia ser considerado como variável do nível da organização. Adotando um delineamento multinível, seria possível testar o efeito do suporte como variável do contexto sobre o bem-estar do trabalhador.

No presente estudo, todas as variáveis foram tratadas no nível individual de análise. O suporte organizacional para ascensão, promoção e salários foi considerado a partir das percepções dos trabalhadores. A maioria dos estudos no campo do bem-estar nas organizações tem se detido no nível individual de análise. Um modelo multinível exige o cumprimento de diversos pressupostos, como o número de organizações participantes, mas esforços deverão ser despendidos pelos pesquisadores organizacionais em estudos futuros.

Por fim, vale ressaltar que, por solicitação das organizações participantes, não puderam ser coletadas e principalmente divulgadas informações adicionais sobre sua estrutura e funcionamento, o que dificultou a utilização de dados sobre a história e a cultura organizacionais para aprofundamento dos achados. O uso de uma abordagem mista (qualitativa e quantitativa) pode oferecer, em pesquisas 
futuras, dados que complementem os resultados publicados na literatura da área até o presente momento.

Artigo recebido em 15.12.2011. Aprovado em 29.07.2012.

\section{Referências}

Abbad, G., \& Torres, C. V. (2002). Regressão múltipla stepwise e hierárquica em Psicologia Organizacional: aplicações, problemas e soluções [Edição Especial]. Estudos de Psicologia, 7, 19-29.

Barbosa, J. G. P, \& Gomes, J. S. (2002). Um estudo exploratório do controle gerencial de ativos e recursos intangíveis em organizações brasileiras. Revista de Administração Contemporânea, 6(2), 29-48. doi: 10.1590/S1415-65552002000200004

Barsky, A., \& Kaplan, S. A. (2007). If you feel bad, it's unfair: A quantitative synthesis of affect and organizational justice perceptions. Journal of Applied Psychology, 92(1), 286-295.

Bass, B. M. (1990). HandBook of leadership: theory, research and managerial aplications. New York: The Free Press.

Bergamini, C. W. (1994). Liderança: administração do sentido. São Paulo: Editora Atlas.

Bergue, S. T. (2010). Gestão de pessoas em organizações públicas. Caxias do Sul, RS: Educs.

Daniels, K. (2000). Measures of five aspects of affective well-being at work. Human Relations, 53(2), 275-294.

Davel, E., \& Machado, H. V. (2001). A dinâmica entre liderança e identificação: sobre a influência consentida nas organizações contemporâneas. Revista de Administração Contemporânea, 5(3), 107-126. doi: 10.1590/S1415-65552001000300006.

Demo, G. (2008). Desenvolvimento e validação da escala de percepção de políticas de gestão de pessoas (EPPGP). Revista de Administração Mackenzie, 9(6), 77-101. doi: 10.1590/S167869712008000600005

Dessen, M. C., \& Paz, M. G (2010). Bem-estar pessoal nas organizações: o impacto de configurações de poder e características de personalidade. Psicologia: Teoria e Pesquisa, 26(3), 549-556.

Diener, E. (1984). Subjective well-being. Psychological Bulletin, 95(3), 542-575. doi: 10.1037/00332909.95.3.542

Dorfman, P. (1996). International and cross-cultural leadership. In B. J. Punnett \& O. Shenkar (Eds.), Handbook for international management research (pp. 267-349). Cambrigde, MA: Blackwell.

Eisenberger, R., Huntington, R., Hutchison, S., \& Sowa, D. (1986). Perceived organizational support. Journal of Applied Psychology, 71(3), 500-507.

Ferraz, R. B., Tavares, H., \& Ziberman, M. L. (2007). Felicidade: uma revisão. Revista de Psiquiatria Clínica, 34(5), 234-242. doi: 10.1590/S0101-60832007000500005

Ferreira, M. C., Silva, A. P., Fernandes, H. A., \& Almeida, S. P. (2008). Desenvolvimento e validação de uma escala de afetos no trabalho (ESAFE). Avaliação Psicológica, 7(2), 143-150. 
Gray, E. K., \& Watson, D. (2001). Emotion, mood, and temperament: similarities, differences, and a synthesis. In R. L. Payne \& C. L. Cooper (Orgs.), Emotions at work: theory, research and applications for management (pp. 21- 43). England: John Wiley \& Sons.

Katz, D., \& Kahn, R. L. (1966). The social psychology of organizations. New York: John Wiley \& Sons.

Kayo, E. K., Kimura, H., Martin, D. M. L., \& Nakamura, W. K. (2006). Ativos intangíveis, ciclo de vida e criação de valor. Revista de Administração Contemporânea, 10(3), 73-90. doi: 10.1590/S1415-65552006000300005

Kelloway, E. K., Gottlieb, B. H., \& Barham, L. (1999). The source, nature, and direction of work and family conflict: a longitudinal investigation. Journal of Occupational Health Psychology, 4(4), 337-346. doi: 10.1037/1076-8998.4.4.337

Lapierre, L. (1989). Imaginário, administração e liderança. Revista de Administração de Empresas, 29(4), 5-16.

Little, L. M., Simmons, B. L., \& Nelson, D. L. (2007). Health among leaders: positive and negative affect, engagement and burnout, forgiveness and revenge. Journal of Management Studies, 44(2), 243-260. doi: 10.1111/j.1467-6486.2007.00687.x

Meleiro, A. R. (2005). Bem-estar no trabalho: os impactos do bem-estar do supervisor e da liderança (Tese de doutorado). Universidade Metodista de São Paulo, São Bernardo do Campo, SP, Brasil.

Melo, E. A. A. (2004). Escala de avaliação do estilo gerencial (EAEG): desenvolvimento e validação. Revista Psicologia: Organizações e Trabalho, 4(2), 31-62.

Mintzberg, H. (1973). The nature of managerial work. New York: Harper \& Row.

O’Driscoll, M. P., \& Beehr, T. A. (1994). Supervisor behaviours, role estressors and uncertainty as predictors of personel outcomes for subordinates. Journal of Organizational Behaviour, 15(2), $141-155$.

Oliveira, P., \& Limongi-França, C. (2005). Avaliação da gestão de programas de qualidade de vida no trabalho. RAE Eletrônica, 4(1), 1-21. Recuperado de http://www.scielo.br/pdf/raeel/v4n1/v4n1a05.pdf. doi: 10.1590/S1676-56482005000100005

Oliveira-Castro, G. A., Pilati, R., \& Borges-Andrade, J. E. (1999). Percepção de suporte organizacional: desenvolvimento e validação de um questionário. Revista de Administração Contemporânea, 3(2), 29-51. doi: 10.1590/S1415-65551999000200003

Paschoal, T., \& Tamayo, A. (2008). Construção e validação da escala de bem-estar no trabalho. Avaliação Psicológica, 7(1), 11-22.

Paschoal, T., Torres, C. V., \& Porto, J. B. (2010). Felicidade no trabalho: relações com suporte organizacional e suporte social. Revista de Administração Contemporânea, 14(6), 1054-1072. doi: $10.1590 / \mathrm{S} 1415-65552010000700005$

Paz, M. G., Gosendo, E. E. M., Dessen, M. C., \& Godeville-Mourão, R. (2009). Justiça organizacional pessoal e bem-estar pessoal nas organizações. Estudos (UCGO), 36(1), 661-687.

Robbins, S. P. (2005). Comportamento organizacional (11a ed.). São Paulo: Pearson Prentice Hall.

Ryan, R. M., \& Deci, E. R. (2001). On happiness and human potentials: a review of research on hedonic and eudaimonic well-being. Annual Review of Psychology, 52(1), 141-166. doi: 10.1146/annurev.psych.52.1.141 
Ryff, C. D., \& Singer, B. H. (2008). Know thyself and become what you are: a eudaimonic approach to psychological well-being. Journal of Happiness Studies, 9(1), 13-39. doi: 10.1007/s10902006-9019-0

Schermerhorn, J. R., Hunt, J. G., Osborn, R. N., \& Uhl-Bien, M. (2010). Organizational behavior (11a ed.). Hoboken, NJ: John Wiley \& Sons.

Seltzer, J., \& Numerof, R. E. (1988). Supervisory leadership and subordinate burnout. Academy of Management Journal, 31(2), 439-446. doi: 10.2307/256559

Silva, A. P. C., \& Ferreira, M. C. (2011). The impact of environmental stressors and types of work contract on occupational stress. The Spanish Journal of Psychology, 14(1), 251-262.

Tamayo, M. R., Pinheiro, F. A., Tróccoli, B. T., \& Paz, M. G. T. (2000, julho). Construção e validação da escala de suporte organizacional percebido (ESOP) [Resumo]. Anais da Reunião Anual da Sociedade Brasileira para o Progresso da Ciência, Brasília, DF. Brasil, 52.

Torres, C. V. (1999). Leadership style norms among americans and brazilians: assessing differences using Jackson's return potencial model (Tese de doutorado). Faculty of the California School of professional Psychology, San Diego, CA, EUA.

Vergara, S. C. (2007). A liderança aprendida. FGV Executivo, 6(1), 61-65.

Warr, P. (2007). Work, happiness and unhappiness. New Jersey: Lawrence Erlbaum Associates.

Waterman, A. S., Schwartz, S. J., \& Conti, R. (2008). The implications of two conceptions of happiness (hedonic enjoyment and eudaimonia) for the understanding of intrinsic motivation. Journal of Happiness Studies, 9(1), 41-79.

Wilson, M. G., DeJoy, D. M., Vandenberg, R., Richardson, H. A., \& McGrath, A. L. (2004). Work characteristics and employee health and well-being: test of a model of healthy work organization. Journal of Occupational and Organizational Psychology, 77(4), 565-588. doi: $10.1348 / 0963179042596522$ 